\title{
BMJ Open What Happens After Menopause? (WHAM): protocol for a prospective, multicentre, age-matched cohort trial of risk-reducing bilateral salpingo- oophorectomy in high-risk premenopausal women
}

\author{
Martha Hickey, ${ }^{1}$ Alison Trainer, ${ }^{2,3}$ Sabine Braat, ${ }^{4}$ Mary-Ann Davey, ${ }^{5}$ \\ Efrosinia Krejany, ${ }^{6}$ John Wark ${ }^{2,7}$
}

To cite: Hickey M, Trainer A, Braat S, et al. What Happens After Menopause? (WHAM): protocol for a prospective, multicentre, age-matched cohort trial of risk-reducing bilateral salpingo-oophorectomy in high-risk premenopausal women. BMJ Open 2017;7:e018758. doi:10.1136/ bmjopen-2017-018758

- Prepublication history for this paper is available online. To view these files, please visit the journal online (http://dx.doi. org/10.1136/bmjopen-2017018758).

Received 20 July 2017 Revised 30 September 2017 Accepted 17 0ctober 2017

CrossMark

For numbered affiliations see end of article.

Correspondence to

Dr Martha Hickey;

hickeym@unimelb.edu.au

\section{ABSTRACT}

Introduction Women at high inherited risk of ovarian cancer are advised to undergo risk-reducing bilateral salpingooophorectomy (RRBSO) at age 40-45 years or when their families are complete. Most women are premenopausal at this age, so RRBS0 will induce surgical menopause. Despite the clear benefits of RRBSO for cancer risk reduction, much less is known about the impact on non-cancer outcomes that contribute to health and well-being and inform surveillance and management strategies.

Methods and analysis This will be a multicentre, prospective cohort study of 105 premenopausal highrisk women undergoing RRBS0 and an age-matched comparison group of 105 premenopausal women not planning oophorectomy or pregnancy in the next 2 years. The aim of this study is to measure the impact of RRBSO on sexual function (primary outcome) at 24 months in highrisk premenopausal women compared with the comparison group. Secondary outcomes include menopausal symptoms and menopause-related quality of life, mood, sleep quality, markers of cardiovascular disease and pre-diabetes, bone density and markers of bone turnover, and the impact of hormone replacement therapy use on these outcomes. Data analysis methods will include logistic and linear regression using general estimating equations accounting for the repeated outcome measurements within each participant. Ethics and dissemination The study has been approved by institutional ethics committees at each participating centre. Findings will be disseminated through peerreviewed publications and conference presentations, and national and international networks of centres managing high-risk women, and will inform national and international clinical guidelines.

Trial registration number The pre-results protocol for this trial is registered with the Australian New Zealand Clinical Trials Registry (anzctr.org.au; registration no: ACTRN12615000082505).

\section{INTRODUCTION}

Ovarian cancer is the fifth most common female cancer and carries a poor prognosis.
Strengths and limitations of this study

- Increasing numbers of women are being diagnosed with gene mutations that increase their risk of ovarian cancer. Currently, risk-reducing bilateral salpingooophorectomy is the only evidence-based intervention to reduce ovarian cancer risk in this population. Since risk-reducing oophorectomy is recommended before age 45 years, it will generally induce surgical menopause.

- Although surgical menopause for risk reduction and other gynaecological indications is relatively common in clinical practice, there have been very few prospective studies of non-cancer outcomes. This will be the largest prospective study of noncancer outcomes following surgical menopause internationally and will provide new evidence to inform patient decision making, clinical management and follow-up protocols.

- A strength of this study is the prospective design and the inclusion of an age-matched control population to account for the impact of age on the outcomes to be studied.

- While the study is adequately powered to address the primary outcome of sexual function, it will have less power to determine the impact of hormone replacement therapy on the outcomes of interest.

- Further potential limitations are the use of hormonal contraceptives (such as the combined oral contraceptive pill) at baseline in participants and the difficulty in recruiting an age-matched control population. To address this we have included multiple and integrated modes of recruitment across a number of centres.

Around $10 \%-15 \%$ of ovarian cancers and over $20 \%$ in women under age 50 are due to germline mutations in the BRCA1 or BRCA2 gene. ${ }^{1}$ These women have an elevated lifetime risk of breast ( $72 \%$ and $69 \%$, respectively) and 
ovarian cancer $\left(44 \%\right.$ and $17 \%$, respectively) ${ }^{2}$ compared with the population risk of $1 \%-5 \%$ for ovarian cancer. Other germline mutations such as the mismatch repair genes responsible for Lynch syndrome increase ovarian cancer risk to around $9 \%{ }^{3}$ The prevalence of germline gene mutations that increase the risk of ovarian cancer is up to 1:400 women, and these cancers commonly develop at an earlier age than in the general population. ${ }^{2}$ The wider availability of rapid, low-cost sequencing methods and the increasing indications for gene testing mean that more women are being diagnosed with germline mutations that increase their risk of ovarian cancer.

There is currently no effective screening strategy for ovarian cancer and the only intervention proven to reduce risk is risk-reducing bilateral salpingo-oophorectomy (RRBSO). Extensive evidence confirms that RRBSO reduces the risk of ovarian cancer by up to $95 \%$ in highrisk women and leads to an overall survival benefit. ${ }^{4}$ RRBSO may also detect occult ovarian cancer. Current guidelines advise RRBSO before aged 40 in BRCA1 carriers or before aged 45 for BRCA2 carriers. ${ }^{5}$

Despite strong evidence that bilateral salpingo-oophorectomy reduces cancer risk and increases survival in high-risk women, many women decline to undergo risk-reducing surgery. Reported uptake rates vary considerably from around $17 \%$ to $89 \%{ }^{6}$ The reasons why many women decline or defer RRBSO are not well understood, but concerns about early menopause are a factor in premenopausal women. ${ }^{7}$ Deleterious gene mutations are commonly identified when women are still premenopausal, so RRBSO will lead to surgical menopause. Despite the clear benefits for cancer risk reduction in high-risk women, very little is known about the impact of RRBSO on non-cancer outcomes, ${ }^{8}$ and this is of concern to high-risk women and their healthcare providers. ${ }^{9}$ In the general population, there is growing evidence that early menopause ( $<45$ years), and particularly surgical menopause, has significant negative consequences for coronary heart disease, ${ }^{10}$ cardiovascular death, all cause-mortality, dementia and Parkinson's disease, particularly for those who do not take hormone replacement therapy (HRT) ${ }^{11}{ }^{12}$ However, the quality of current evidence is low, and there have been very few prospective studies of surgical menopause in any population.

High-risk women considering RRBSO are faced with complex decision making, weighing up the potential adverse health implications of bilateral oophorectomy against the known reduction in cancer risk. ${ }^{5}$ Most studies of RRBSO have focused exclusively on cancer outcomes, but current evidence suggests that premenopausal women experience a significant worsening of vasomotor symptoms (hot flushes and night sweats), a persistent decline in menopause-related quality of life (QOL) and sexual function 1 year after RRBSO ${ }^{13}$ HRT partly mitigates these symptoms, but even in HRT users sexual function and vaginal symptoms do not return to presurgical levels. ${ }^{14}{ }^{15}$ Use of HRT is thought to be safe in high-risk women, provided they do not have a personal history of breast cancer, but the proportion of users is unknown and evidence for safety is limited. ${ }^{16}$

Bilateral salpingo-oophorectomy leads to infertility, and women may consider undergoing concurrent hysterectomy. Hysterectomy adds to the duration, cost and potential complications of surgery, but avoids the need for combined HRT and removes the risk of endometrial pathology associated with tamoxifen use. ${ }^{17}$ More information is needed from prospective studies to inform women and their healthcare providers considering hysterectomy at the time of RRBSO.

To support informed decision making and to appropriately structure follow-up care, there is an unmet need for prospective data on the non-cancer consequences of RRBSO. ${ }^{5}$ Having reduced their risks of breast and ovarian cancer, high-risk women should reasonably anticipate a normal QOL and life expectancy. In order to decide whether and when to undergo RRBSO, and how best to optimise health postoperatively, more information is needed about the non-cancer consequences of risk-reducing oophorectomy.

This multicentre, population-based, controlled, cohort study will generate new data to inform decision making around RRBSO and evidence-based follow-up care in the general population following surgical menopause. The primary objective of WHAM (What Happens After Menopause) is to assess the association between RRBSO and sexual function as measured by the Female Sexual Function Index (FSFI). The secondary outcomes are menopausal symptoms and menopause-related QOL, mental health, bone health and turnover, cardiometabolic risk, and sleep quality. We hypothesise that RRBSO in premenopausal women will reduce sexual function (primary outcome), increase menopausal symptoms, reduce menopause-related QOL, increase bone turnover, reduce bone density, increase cardiometabolic risk and reduce sleep quality compared with age-matched premenopausal women who retain their ovaries. We do not expect RRBSO to affect mental health. ${ }^{18}$

\section{METHODS: PARTICIPANTS AND OUTCOMES}

\section{Study design and setting}

This is a multicentre cohort study comparing 105 highrisk premenopausal women up to 50 years of age who plan to undergo RRBSO with 105 age-matched, premenopausal women who do not plan to undergo oophorectomy or pregnancy in the next 2 years. Study recruitment commenced in April 2013 and is ongoing, enrolling women from eight public and four private hospitals in Australia (Victoria and New South Wales). The projected timeline for recruitment is 3-4 years. All subjects will be followed up for 2 years from the baseline visit.

\section{Eligibility criteria and recruitment}

Eligible women will be premenopausal and age up to 50 years with regular menstrual periods (if intact uterus), no vasomotor symptoms and a follicular stimulating 
hormone level of $<15$ IU $/ \mathrm{L}$ on days $2-6$ of the menstrual cycle. Exclusions include $<3$ months since pregnancy and lactation, abnormal uterine bleeding, or use of antioestrogens such as tamoxifen.

Exposed women are defined as those at high inherited risk of ovarian cancer due to confirmed presence of a gene mutation (carriers of BRCA1, BRCA2, BRIP1, RAD51C, Lynch syndrome) or high familial risk of ovarian cancer and planning to undergo RRBSO. Those with a personal history of breast cancer will be included if they remain premenopausal and are not currently taking endocrine therapy. Participants will be recruited via clinician referrals from familial cancer, high-risk breast cancer and menopause clinics, referrals from public and private gynaecology oncologists and surgeons, and via targeted advertising through mainstream and electronic media (eg, newspaper and television reports, cancer registries, cancer support newsletters and websites). Each referral will be processed on receipt and eligibility screening and subsequent recruitment will be scheduled to occur within 8 weeks prior to the RRBSO surgery date. The baseline visit will be scheduled for the early follicular phase (days 2-6) of the subject's menstrual cycle prior to RRBSO.

Women in the comparison group are defined as 1:1 individually age-matched (within \pm 5 years), premenopausal women who are not planning oophorectomy or pregnancy within the next 2 years. Comparison subjects can be low-risk or relatives of high-risk women who do not carry a gene mutation. The comparison group will be recruited via mainstream and electronic media advertising to the general public (eg, clinical research recruitment websites, hospital and university staff newsletters and websites), and by asking recruited cases to recommend the study to relatives and friends. Recruitment will be scheduled to occur during the early follicular phase (days 2-6) of the subject's menstrual cycle, and within 8 weeks after eligibility screening. Women in the comparison group withdrawing prior to the 6-month follow-up will be replaced by women of similar baseline age.

\section{Participant timeline}

A schedule of study assessments and measurements is presented in table 1. After informed consent and eligibility, baseline data will be collected including obstetric, gynaecological, medical and surgical history, current medications, risk factors for fracture (www.shef.ac.uk/ FRAX), risk factors for cardiovascular disease including blood pressure, circulating cholesterol and lipids and $\mathrm{C}$ reactive protein (www.heartfoundation.org.au/SiteCollectionDocuments/austcardiovascular-risk-charts.pdf), tobacco, drug and alcohol use, methods of contraception, methods of breast cancer surveillance, germline mutation type, and family cancer history. In those with a history of breast cancer, stage and grade of tumour and treatment history will be recorded. Blood pressure, weight, height and waist-hip ratio will be measured and repeated at selected follow-up visits (see table 1). A blood sample will be taken after an overnight fast to measure sex steroid concentrations, lipids and cardiovascular risk factors, which will be repeated at 1-year and 2-year follow-up. A urine sample will be collected for comparison subjects to exclude pregnancy (see table 1). Medications used in the 3 months prior to enrolment and throughout the 2-year follow-up period will be recorded and include those related to bone health (calcium, vitamin $\mathrm{D}$ and antiresorptive agents), depression, anxiety, HRT, non-hormonal treatments for vasomotor symptoms, contraception and insomnia. Adverse events (AEs) (changes in physical and psychological health from baseline) will be monitored over the 2-year follow-up period. Questionnaires to measure sexual function, menopausal symptoms, menopause-related QOL, sleep quality, depression and anxiety will be administered at baseline and all postbaseline study visits (see table 1). All participants will undergo DXA scans within 3 months of RRBSO (baseline) and again at 1 and 2years postbaseline.

Participants will be free to withdraw at any time, either through formal revocation of consent (patient preference) or via ceased communication with the recruitment site (lost to follow-up). All collected data will be included in the analyses and any discrepancies related to data collected prior to withdrawal will be queried with the subject up to 1 month after the withdrawal date. In order to minimise missing data, participants who cannot complete all scheduled visits will be invited to continue in the study despite missing visits. Those who withdraw at a scheduled time-point or who are unable to attend the recruitment site at a scheduled time-point will be offered the opportunity to complete any time-point data that can be captured remotely via telephone and email correspondence, including questionnaire, medications and $\mathrm{AE}$ data.

\section{Outcomes}

The primary outcome is the change in sexual function following RRBSO, as measured by the FSFI. ${ }^{19}$ Sexual function was selected because it is a patient priority and in young women and because previous prospective studies suggest that sexual function may be permanently impaired following RRBSO. ${ }^{13-15}$ Secondary outcomes include menopause-related QOL, menopausal symptoms, sleep quality, depression and anxiety, markers of cardiovascular disease and pre-diabetes, and bone density and markers of bone turnover. All measures will be collected at baseline (prior to RRBSO in the intervention group) with follow-up measures scheduled as per table 1 .

\section{Measurement of sexual function}

Subjects will be asked whether they currently have a sexual partner and whether sexual problems for the partner impact on their sexual activity. We will use standardised and validated questionnaires to measure three aspects of sexual function:

1. The FSFI is a widely used brief self-report questionnaire that assesses six separate dimension: desire, arousal, lubrication, orgasm, satisfaction 
Table 1 Schedule of assessments/investigations

\begin{tabular}{|c|c|c|c|c|c|}
\hline Study procedures & $\begin{array}{l}\text { Baseline } \\
\text { Day 1* } \\
\text { (visit 1) }\end{array}$ & $\begin{array}{l}\text { Month } 3 \\
\pm 6 \text { weeks } \\
\text { (visit 2) }\end{array}$ & $\begin{array}{l}\text { Month } 6 \\
\pm 6 \text { weeks } \\
\text { (no visit) }\end{array}$ & $\begin{array}{l}\text { Month } 12 \\
\pm 6 \text { weeks } \\
\text { (visit 3) }\end{array}$ & $\begin{array}{l}\text { Month } 24 \\
\pm 6 \text { weeks } \\
\text { (visit 4) }\end{array}$ \\
\hline Informed consent and eligibility & $\bullet$ & & & & \\
\hline $\begin{array}{l}\text { Surgical, medical, gynaecological, obstetric and menstrual } \\
\text { history }\end{array}$ & $\bullet$ & & & & \\
\hline Smoking, drug and alcohol use history & • & & & & \\
\hline Germline mutation testing history & $\bullet$ & & & & \\
\hline Personal and family cancer history & $\bullet$ & & & & \\
\hline $\begin{array}{l}\text { Personal breast cancer surveillance, diagnosis, treatment } \\
\text { details (if applicable) }\end{array}$ & $\bullet$ & & & & \\
\hline Contraceptive methods & • & & & & \\
\hline $\begin{array}{l}\text { Fracture risk assessment† } \\
\text { (http://www.shef.ac.uk/FRAX/tool.jsp) }\end{array}$ & $\bullet$ & & & & \\
\hline Height & $\bullet$ & & & & \\
\hline Weight & $\bullet$ & & & - & $\bullet$ \\
\hline Waist-hip ratio (http://bupa.com.au/) & $\bullet$ & & & $\bullet$ & $\bullet$ \\
\hline Blood pressure & $\bullet$ & $\bullet$ & & $\bullet$ & $\bullet$ \\
\hline Urinary pregnancy test (comparisons only) & $\bullet$ & $\bullet$ & & $\bullet$ & - \\
\hline Medications & $\bullet$ & $\bullet$ & $\bullet$ & $\bullet$ & $\bullet$ \\
\hline Adverse events & & $\bullet$ & - & - & - \\
\hline \multicolumn{6}{|l|}{ Questionnaires } \\
\hline $\begin{array}{l}\text { FSFI, FSDS-R and SAQ (sexual function, distress and } \\
\text { activity) }\end{array}$ & - & $\bullet$ & $\bullet$ & $\bullet$ & - \\
\hline $\begin{array}{l}\text { Green Climacteric Scale and MENQOL (menopausal } \\
\text { symptoms and QOL) }\end{array}$ & $\bullet$ & $\bullet$ & $\bullet$ & - & - \\
\hline PSQI (sleep quality) & $\bullet$ & - & - & - & - \\
\hline CES-D (depression) and GAD-7 (anxiety) & $\bullet$ & $\bullet$ & $\bullet$ & $\bullet$ & $\bullet$ \\
\hline \multicolumn{6}{|l|}{ Blood tests (fasting) } \\
\hline FSH, oestradiol (days 2-6 menstrual cycle) & $\bullet$ & & & & \\
\hline $\begin{array}{l}\text { Total testosterone, Dehydroepiandrosterone, } \\
\text { Androstenedione, SHBG }\end{array}$ & $\bullet$ & & & $\bullet$ & • \\
\hline $\begin{array}{l}\text { Total cholesterol, LDL-C, HDL-C, triglycerides, insulin, } \\
\text { glucose, HbA1c, CRP }\end{array}$ & $\bullet$ & & & - & • \\
\hline $\begin{array}{l}\text { Albumin, creatinine, calcium, phosphate, P1NP, BCTX, } \\
\text { parathyroid hormone, vitamin D }\end{array}$ & $\bullet$ & & & $\bullet$ & - \\
\hline \multicolumn{6}{|l|}{ Bone mineral density (BMD) } \\
\hline $\begin{array}{l}\text { DXA scans of hip and lumbar spine BMD and of total body } \\
\text { bone mineral content }\end{array}$ & & $\bullet$ & & - & - \\
\hline
\end{tabular}

*The baseline visit will be performed up to 8 weeks prior to risk-reducing bilateral salpingo-oophorectomy for the intervention group, or following eligibility screening for controls.

†The FRAX tool will only be applied to women aged over 40 years at baseline.

CES-D, Center for Epidemiologic Studies Depression; CRP, C reactive protein; DXA, dual-energy X-ray absorptiometry; FSDS-R, Female Sexual Distress Scale; FSFI, Female Sexual Function Index; FSH, follicular stimulating hormone; GAD-7, Generalised Anxiety Disorder; $\mathrm{HbA1c}$, haemoglobin A1c; HDL-C, high-density lipoprotein; LDL-C, low-density lipoprotein; MENQOL, Menopause-Related Quality of Life; PSQI, Pittsburgh Sleep Quality Index; QOL, quality of life; SAQ, Sexual Activity Questionnaire; SHBG, sex hormone binding globulin.

and pain, and it also provides a total score. ${ }^{20}$ The FSFI has been validated in a large group of women with sexual arousal disorder versus age-matched controls ${ }^{19}$ and in cancer survivors. ${ }^{21}$ It has demonstrated a high level of acceptability, reliability and validity in both cancer and non-cancer populations. ${ }^{22}$ The FSFI has a high internal consistency and test-retest reliability, and differentiates well between sexually dysfunctional and non-dysfunctional women, and is highly sensitive in discriminating between clinical and non-clinical 
populations. ${ }^{20}$ FSFI scores (primary) will indicate which domains of sexual function are affected by RRBSO. The FSFI takes $<5$ min to complete.

2. The revised Female Sexual Distress Scale (FSDS-R) ${ }^{23}$ is a brief (13 items) questionnaire that measures the extent to which reduced sexual desire causes distress in women. Distress is a key feature of hypoactive sexual desire disorder (HSDD). The FSDS-R has good discriminant validity, test-retest reliability and internal consistency in measuring sex-related personal distress in women with HSDD. ${ }^{24}$ The scale also has good content validity (relevance, clarity, comprehensiveness) ${ }^{25}$

3. The Sexual Activity Questionnaire (SAQ) evaluates sexual pleasure (desire, enjoyment and satisfaction), discomfort (vaginal dryness and dyspareunia) and habit (frequency of sexual activity compared with usual activity). ${ }^{26}$ The SAQ has been validated in patients with breast cancer and in high-risk women. ${ }^{27}$ It is quick and easy to administer and has good face validity discriminating between the sexual functioning of premenopausal and postmenopausal women ${ }^{27}$

\section{Measurement of circulating testosterone and SHBG}

Circulating testosterone concentrations are reduced following RRBSO, and this may contribute to sexual function, although findings are conflicting. ${ }^{15}$ Circulating concentrations of total testosterone and the testosterone precursors, androstenedione and 5-dehydroepiandrosterone (DHEA), and sex hormone binding globulin (SHBG) will be measured at baseline and follow-up, as per table 1. Fasting blood samples will be immediately centrifuged to isolate plasma, and stored at $-80^{\circ} \mathrm{C}$. Because conventional radioimmunoassays lack sensitivity at low androgen concentrations found after surgical menopause, ${ }^{28}$ we will use liquid chromatography tandem mass spectrometry (LC-MS/MS), using a Sciex API 5500Q instrument by CPR Pharma Services (Adelaide, South Australia, Australia). The lower limit of measurement is $0.025 \mathrm{ng} / \mathrm{mL}$ for testosterone, $0.05 \mathrm{ng} / \mathrm{mL}$ for androstenedione and $0.5 \mathrm{ng} / \mathrm{mL}$ for DHEA. The intra-assay coefficients of variation are low $(<5 \%)$ at $1 \mathrm{nM}$. SHBG concentrations will be measured using a non-competitive liquid-phase Radio-immuno assay (68562, Orion Diagnostica, Espoo, Finland). We will calculate free testosterone concentrations using measured total testosterone and SHBG concentrations. ${ }^{28}$

\section{Measurement of menopausal symptoms and menopause- related QOL}

Menopausal symptoms will be measured using the Greene Climacteric Scale, which measures the frequency and severity of menopausal symptoms in psychological somatic and vasomotor domains. ${ }^{29}$ The scale is widely used and has been validated in an Australian population. ${ }^{30}$

Menopause-related QOL will be measured using the Menopause-Related Quality of Life Intervention (MENQOL-Intervention) questionnaire. ${ }^{31}$ The MENQOL-Intervention covers four domains-vasomotor, physical, psychosocial and sexual-and includes a global QOL item. It is widely used internationally and its strength and validity have recently been confirmed in a large population-based sample of midlife women ${ }^{32}$ and in breast cancer survivors. ${ }^{33}$ The questionnaire tests the impact of an intervention, such as oophorectomy, on symptoms. Each takes less than 5 min to complete. Use of HRT and of non-hormonal medications for vasomotor symptoms will be recorded, and subjects will be asked about decision making around HRT.

\section{Measurement of sleep quality}

Sleep quality will be measured using the Pittsburgh Sleep Quality Index (PSQI), ${ }^{34}$ a widely used and validated measure of sleep quality. ${ }^{35}$ The questionnaire measures subjective sleep quality, latency, duration, habitual sleep efficiency, sleep disturbances and medications, and daytime dysfunction. The PSQI takes $<5$ min to complete.

\section{Measurements of depression and anxiety}

Depression and anxiety will be measured using the Center for Epidemiologic Studies Depression (CES-D) ${ }^{36}$ and the Generalised Anxiety Disorder (GAD-7) ${ }^{37}$ scales, respectively. The CES-D measures the frequency of depressive feelings and behaviours experienced in the past week and includes 20 items that are assigned scores ranging from 0 to 3. The final CES-D score $(0-60)$ is the sum of the 20 items and a score of $\geq 16$ points is indicative of depression. The GAD-7 measures the frequency of GAD symptoms in the past fortnight and includes seven items that are assigned scores ranging from 0 to 3 . The final GAD-7 score $(0-21)$ is the sum of the seven items and scores of $5-9,10-14$ and 15-21 are indicative of mild, moderate and severe anxiety, respectively. Each questionnaire takes $<5$ min to complete.

\section{Measurement of cardiovascular disease risk}

Resting blood pressure, weight, height and waist/hip ratio will be measured as per table 1. Fasting blood samples will be analysed for serum insulin, glucose, triglycerides, cholesterol, high-density lipoprotein (HDL-C), low-density lipoprotein (LDL-C) and high-sensitivity $\mathrm{C}$ reactive protein, which predicts cardiovascular disease in healthy women. ${ }^{38}$ These have not previously been prospectively measured in surgical menopause. Homeostasis model assessment (HOMA) will be calculated by fasting insulin $(\mu \mathrm{U} / \mathrm{mL}) \times$ fasting glucose $(\mathrm{mM}) / 22.5$. Insulin resistance will be defined by a HOMA reading $>488$. The presence of diabetes or pre-diabetes will be established by fasting glucose and haemoglobin A1c (HbA1c). An HbAlc of $>6.5 \%$ will be used as a cut-off for the diagnosis of diabetes as recommended by the American Diabetes Association (www.diabetes.org) and carried out in National Association of Testing Authorities accredited laboratories using standard equipment.

Measurement of bone density and markers of bone turnover We will measure fasting serum albumin, creatinine, calcium and phosphate, and circulating markers of 
bone turnover (beta $\mathrm{C}$ terminal telopeptide of type I collagen (beta CTX) and N terminal propeptide of type 1 collagen) ${ }^{39}$ We will measure $25-\mathrm{OH}$ vitamin $\mathrm{D} 2$ and D3 using LC-MS/MS and intact parathyroid hormone immunoassay. The bone turnover markers are independent predictors of fracture risk and will supplement bone mineral density (BMD) data. Regional BMD (at the lumbar spine and hip) will be measured using dual-energy X-ray absorptiometry (DXA) within 3 months of RRBSO (baseline). We will also use DXA to measure total body bone mineral content, which will increase power to detect bone loss. ${ }^{40}$ DXA measurement location will be included as a covariate and change from baseline will be used in outcome analyses. Fracture risk assessment using a FRAX tool (www.shef.ac.uk/FRAX) will only be applied to women aged over 40 years.

\section{Sample size}

We will recruit 105 high-risk premenopausal women planning RRBSO and 105 age-matched ( \pm 5 years) women in the comparison group. The primary outcome is sexual dysfunction (FSFI score <26.55), ${ }^{41}$ where lower FSFI scores indicate worse sexual function. Based on large population-based surveys, we have assumed that around $24 \%$ of premenopausal women will have sexual dysfunction (FSFI below clinical cut-off) at baseline, ${ }^{42}{ }^{43}$ and that the proportion with sexual dysfunction does not differ between the high-risk women or those in the comparison group, or between age groups, and that it does not change over the 2-year follow-up period in the comparison group. Sample size calculations are based on comparing the proportion of women with sexual dysfunction in the RRBSO group with the comparison group at the 2-year follow-up. A sample size of 89 women per group will provide $80 \%$ power at a two-sided 5\% level of significance to detect a $21 \%$ difference (from $24 \%$ in the comparison group to $45 \%$ in the RRBSO group), or 105 per group when allowing for $15 \%$ loss to follow-up at 2 years. This difference in the proportion of women with sexual dysfunction is clinically significant. ${ }^{44}$ For the secondary outcomes, this sample size of 89 per group will also provide $83 \%$ power to detect a difference in the proportion of women with a DXA T-score of $\leq-1$ at 2 years, from $16 \%$ in the comparison group to $36 \%$ in the RRBSO group, ${ }^{45}$ and $92 \%$ power to detect a mean difference between groups of $0.5 \mathrm{SD}$ in the MENQOL score.

\section{METHODS: DATA COLLECTION, MANAGEMENT AND ANALYSIS Data collection methods}

Study data are being collected using paper case report forms, paper self-reported questionnaires, paper recorded phone interviews, and paper copies of biochemical, pathology and bone density imaging reports. These paper documents will be kept by each recruitment site in a locked cabinet accessible to local research staff only. All data will be entered and stored in a de-identified, password-protected electronic study database created with the Research Electronic Data Capture (REDCap) web application (https://projectredcap.org/). ${ }^{44}$ REDCap is a secure, web-based application designed to support data capture for research studies, providing (1) an intuitive interface for validated data entry; (2) audit trails for tracking data manipulation and export procedures; (3) automated export procedures for seamless data downloads to common statistical packages; and (4) procedures for importing data from external sources. ${ }^{46}$ Internet access to the REDCap database will use a secure server located at the University of Melbourne, Australia (REDCap consortium host), and access will be limited to local research staff at each recruitment site. Research staff will only have access to the REDCap data collected at their local recruitment site, whereas the chief investigator, project manager, data administrator and statistician will have access to all REDCap data.

\section{Data management}

Management of comprehensive and valid paper records will be the responsibility of local research staff at each recruitment site. To ensure a systematic approach to data collection, the project manager will provide protocol training and written standard operating procedures to research staff prior to any study visits being performed. Data integrity will be maintained through review of all collected data prior to, during and after each study visit. Any time-sensitive data (eg, questionnaires) will be reviewed as soon as they are collected and any identified discrepancies will be resolved within 1 week of collection. Medications and $\mathrm{AE}$ data will be reviewed and consolidated at every study visit. All other data discrepancies will be queried with the subject via email or telephone correspondence and resolved within 1 month of collection. Records of participant screenings and enrolments at each site will be maintained using Microsoft Excel spreadsheets with diary features. These documents will assist research staff to adhere to study timelines and to regularly monitor and report site study progress to the project manager, investigators and the responsible Human Research Ethics Committees. Electronic data entry into the REDCap database will begin no later than 12 months prior to the last participant last visit. Database training of research staff and data entry will be conducted and overseen (respectively) by the project manager.

\section{Data and safety monitoring}

We do not anticipate any study-related AEs or serious adverse events. The only anticipated AEs include phlebotomy-related site injuries (minor) and vasovagal syncope (rarely), and negative psychological responses to questionnaires and interview discussions. These AEs will be circumvented or managed by providing the subject with appropriate advice and counselling during and after each study visit. Reports of biochemistry, pathology and bone density results will be routinely reviewed by medically qualified site investigators. Any concerning results will be immediately reviewed and reported to the subject 
and her general practitioner (GP). All other results will be reviewed within 1 week of report receipt and any results outside of normal ranges will be reported to the subject's GP within 2 weeks. The project manager will directly monitor all Victorian data within 1 month of collection, and personally visit other recruitment sites on a 6-monthly basis to perform data monitoring activities. The outcomes will be reported to the chief investigator and all relevant research staff and investigators within 1 month of each monitoring visit, and any systematic problems with data collection, data management and $\mathrm{AE}$ monitoring and reporting will be rectified. Questionnaires will be independently scored on two separate occasions prior to data entry into the REDCap database, and all data entry into REDCap will be monitored by an independent data administrator. REDCap data queries will be addressed by the project manager and data administrator using the REDCap Data Resolution Workflow module.

\section{Statistical methods}

The analysis set will consist of all recruited women. Baseline characteristics will be summarised by group. The primary outcome is sexual dysfunction, defined as an FSFI score of $<26.55$ at 2 years. The primary outcome and binary secondary outcomes (eg, PSQI score $>5$, CES-D score $\geq 16$, GAD-7 $\geq 10$, absolute change from baseline in diastolic blood pressure $\geq 5 \mathrm{~mm} \mathrm{Hg}$, relative change from baseline in HDL:LDL ratio $>2.5$, and BMD T-score $\leq$ -1 ) will be analysed by fitting a generalised linear model with a logistic link function using generalised estimation equations to account for the repeated measurements. ${ }^{47}$ Continuous secondary outcomes including FSFI as a continuous outcome will be analysed by fitting a linear regression model using generalised estimating equations. The matching variable age group will be included in the above models. Appropriate transformation of a continuous variable may be performed before analyses if the variable is found to be skewed. A directed acyclic graph will be used to explore which covariates to include in the adjusted -model. ${ }^{48}{ }^{49}$ An adjusted model will be fitted to correct for the potential confounding of time-independent covariates (eg, use of hormonal contraception at baseline, use of antidepressant medication at baseline) and time-dependent covariates (eg, symptoms of depression and/or anxiety). We anticipate that around one-third of women will have concurrent hysterectomy at the time of RRBSO, that $40 \%$ will take HRT following RRBSO, and that $10 \%$ of high-risk women will either have a history of breast cancer or will develop breast cancer over the follow-up period. In addition to the primary comparison of all RRBSO participants with all controls, we will compare outcomes between the three subgroups (RRBSO \pm hysterectomy, \pm HRT, \pm breastcancer) with all controls.

The number and proportion of women using specific medication (eg, HRT, antidepressants) will be summarised by group. AEs will be recorded and graded using the revised National Cancer Institute Common Terminology
Criteria for Adverse Events criteria, and the number and proportion of women with at least one $\mathrm{AE}$ will be summarised by group. Exploratory subgroup analyses will examine whether the effect differs across the following subgroup categories: ( 1 ) age at baseline ( $<45$ years vs $\geq 45$ years) and (2) use of hormonal contraception at baseline (yes vs no) by adding the subgroup and its interaction with group to the model.

\section{Outcomes and significance}

Growing numbers of women are known to be at high inherited risk of ovarian cancer and are electing to undergo RRBSO. The established reduction in ovarian cancer mortality following RRBSO could be further improved by minimising the negative non-cancer consequences, which also may contribute to morbidity and all-cause mortality. This can be addressed only when the non-cancer outcomes are known, and this requires prospective data collection. The principal significance is high burden of disease from ovarian cancer and the growing number of high-risk women who would benefit from RRBSO. In addition, because prospective studies of surgical menopause in the general population are very limited, the findings from this study will also inform care of low-risk women undergoing surgical menopause. These data will be used to develop resources to support decision making around RRBSO, including the timing of surgery, additional hysterectomy, preoperative risk assessment and counselling, and postoperative follow-up including use of HRT. Our studies will directly improve cancer outcomes by impact on clinical practice and policy, through the development of multidisciplinary evidence-based guidelines and screening protocols. Consumers have clearly indicated that they wish to be informed about the consequences of RRBSO and that current information provision and follow-up care are inadequate.$^{50}{ }^{51}$ Decision making and satisfaction are improved when high-risk women are offered dedicated clinical services. ${ }^{52} 53$ Our data and guidelines will provide a template for the care of other high-risk women considering RRBSO as new genes are identified. Improved information about the non-cancer consequences of RRBSO is unlikely to dissuade highrisk women from surgery. Despite side effects, most do not regret their choice. ${ }^{18}{ }^{50}$ Improved follow-up care may also improve QOL for high-risk women and contribute to early detection and/or prevention of conditions related to early menopause. This will be the first international multicentre, prospective study of non-cancer outcomes after RRBSO or surgical menopause in any population. Currently, there is no consensus on how high-risk women should be managed following RRBSO. This study will provide new evidence on which to develop evidencebased care for this growing population of women.

\section{ETHICS AND DISSEMINATION}

This is a prospective observational cohort study of highrisk women undergoing RRBSO. No study-related safety 
concerns are anticipated. The study has been granted ethics approval at each of the participating recruitment centres.

Dissemination will be through peer-reviewed publications, presentations at national and international conferences, and existing networks including the Women Choosing Surgical Prevention trial, national and international menopause societies, and specialist colleges.

\section{Author affiliations}

${ }^{1}$ Department of Obstetrics and Gynaecology, The Royal Women's Hospital, University of Melbourne, Melbourne, Victoria, Australia

${ }^{2}$ Department of Medicine, University of Melbourne, Melbourne, Victoria, Australia ${ }^{3}$ Parkville Familial Cancer Centre, Peter MacCallum Cancer Centre, Melbourne, Victoria, Australia

${ }^{4}$ Melbourne Clinical and Translational Sciences (MCATS), Melbourne School of Population and Global Health, University of Melbourne, Melbourne, Victoria, Australia ${ }^{5}$ Department of Obstetrics and Gynaecology, Monash University, Melbourne, Victoria, Australia

${ }^{6}$ Gynaecology Research Centre, The Royal Women's Hospital, Parkville, Victoria, Australia

${ }^{7}$ Department of Bone and Mineral Medicine, Royal Melbourne Hospital, University of Melbourne, Melbourne, Victoria, Australia

Acknowledgements The authors would like to thank clinicians at the recruiting sites: Dr Judy Kirk, Associate Professor Alison Brand, Dr Lesley Andrews, Associate Professor Trevor Tejana-Berges and Dr David Wrede.

Contributors MH, AT, SB, M-AD, EOK and JW contributed to the conception, design, writing and editing of the protocol. $\mathrm{MH}$ drafted the manuscript, and all other authors contributed to the final version. SB wrote the statistical methods. MH is the guarantor. All authors read and approved the final manuscript.

Funding The study is supported by an NHMRC Project Grant (APP1048023) and by the Australia and New Zealand Gynaecological Oncology Group. MH is supported by an NHMRC Practitioner Fellowship (APP1058935).

Competing interests None declared.

Patient consent We are using Peter MacCallum Cancer Centre HREC approved consent forms.

Ethics approval The study has been granted ethics approval at each of the participating recruitment centres, including the following: Peter MacCallum Cancer Centre (Victoria, Australia), HREC/12/PMCC/24, 12/90, 04/12/2012; The Royal Women's Hospital (Victoria, Australia), HREC/12/PMCC/24, 12/07, 20/12/2012; The Royal Melbourne Hospital (Victoria, Australia), HREC/12/PMCC/24, 2013/060, 07/10/2013; Prince of Wales Hospital) (New South Wales, Australia), HREC/13/ POWH/61, 12/304, 23/05/2013; Westmead Hospital (New South Wales, Australia), HREC/13/POWH/61, SSA/13/WMEAD/189, 28/11/2013; Royal Hospital for Women (New South Wales, Australia), HREC/13/POWH/61, 14/G/146, 29/04/2014; Royal Prince Alfred Hospital (New South Wales, Australia), X14-0396, 10/21, 22/04/2015; and Chris 0'Brien Lifehouse (New South Wales, Australia), X14-0396, LH15/035, 10/08/2015.

Provenance and peer review Not commissioned; externally peer reviewed.

Open Access This is an Open Access article distributed in accordance with the Creative Commons Attribution Non Commercial (CC BY-NC 4.0) license, which permits others to distribute, remix, adapt, build upon this work non-commercially, and license their derivative works on different terms, provided the original work is properly cited and the use is non-commercial. See: http://creativecommons.org/ licenses/by-nc/4.0/

(C) Article author(s) (or their employer(s) unless otherwise stated in the text of the article) 2017. All rights reserved. No commercial use is permitted unless otherwise expressly granted.

\section{REFERENCES}

1. Alsop K, Fereday S, Meldrum C, et al. BRCA mutation frequency and patterns of treatment response in BRCA mutation-positive women with ovarian cancer: a report from the Australian Ovarian Cancer Study Group. J Clin Oncol 2012;30:2654-63.

2. Kuchenbaecker KB, Hopper JL, Barnes DR, et al. Risks of breast, ovarian, and contralateral breast cancer for BRCA1 and BRCA2 mutation carriers. JAMA 2017;317:2402-16.

3. Bonadona V, Bonaïti B, Olschwang S, et al. Cancer risks associated with germline mutations in MLH1, MSH2, and MSH6 genes in Lynch syndrome. JAMA 2011;305:2304-10.

4. Marchetti C, De Felice F, Palaia I, et al. Risk-reducing salpingooophorectomy: a meta-analysis on impact on ovarian cancer risk and all cause mortality in BRCA 1 and BRCA 2 mutation carriers. BMC Womens Health 2014;14:150.

5. Hartmann LC, Lindor NM. The role of risk-reducing surgery in hereditary breast and ovarian cancer. N Engl J Med 2016;374:454-68.

6. Sidon L, Ingham S, Clancy T, et al. Uptake of risk-reducing salpingooophorectomy in women carrying a BRCA1 or BRCA2 mutation: evidence for lower uptake in women affected by breast cancer and older women. Br J Cancer 2012;106:775-9.

7. Mai PL, Piedmonte M, Han PK, et al. Factors associated with deciding between risk-reducing salpingo-oophorectomy and ovarian cancer screening among high-risk women enrolled in GOG-0199: an NRG Oncology/Gynecologic Oncology Group study. Gynecol Oncol 2017;145:122-9.

8. Cohen JV, Chiel L, Boghossian L, et al. Non-cancer endpoints in BRCA1/2 carriers after risk-reducing salpingo-oophorectomy. Fam Cancer 2012;11:69-75.

9. Miller SM, Roussi P, Daly MB, et al. New strategies in ovarian cancer: uptake and experience of women at high risk of ovarian cancer who are considering risk-reducing salpingo-oophorectomy. Clin Cancer Res 2010;16:5094-106.

10. Mytton J, Evison F, Chilton PJ, et al. Removal of all ovarian tissue versus conserving ovarian tissue at time of hysterectomy in premenopausal patients with benign disease: study using routine data and data linkage. BMJ 2017;356:j372.

11. Rocca WA, Gazzuola-Rocca L, Smith CY, et al. Accelerated accumulation of multimorbidity after bilateral oophorectomy: a population-based cohort study. Mayo Clin Proc 2016;91:1577-89.

12. Evans EC, Matteson KA, Orejuela FJ, et al. Salpingo-oophorectomy at the time of benign hysterectomy: a systematic review. Obstet Gynecol 2016:128:476-85.

13. Finch A, Metcalfe KA, Chiang JK, et al. The impact of prophylactic salpingo-oophorectomy on menopausal symptoms and sexual function in women who carry a BRCA mutation. Gynecol Oncol 2011;121:163-8.

14. Johansen $N$, Liavaag $A H$, Tanbo TG, et al. Sexual activity and functioning after risk-reducing salpingo-oophorectomy: Impact of hormone replacement therapy. Gynecol Oncol 2016;140:101-6.

15. Tucker PE, Bulsara MK, Salfinger SG, et al. Prevalence of sexual dysfunction after risk-reducing salpingo-oophorectomy. Gynecol Oncol 2016;140:95-100.

16. Domchek S, Kaunitz AM. Use of systemic hormone therapy in BRCA mutation carriers. Menopause 2016;23:1026-7.

17. Shu CA, Pike MC, Jotwani AR, et al. Uterine Cancer after riskreducing salpingo-oophorectomy without hysterectomy in women With BRCA mutations. JAMA Oncol 2016;2:1434-40.

18. Finch A, Metcalfe KA, Chiang J, et al. The impact of prophylactic salpingo-oophorectomy on quality of life and psychological distress in women with a BRCA mutation. Psychooncology 2013;22:212-9.

19. Rosen R, Brown C, Heiman J, et al. The Female Sexual Function Index (FSFI): a multidimensional self-report instrument for the assessment of female sexual function. J Sex Marital Ther 2000;26:191-208.

20. Meston CM, Derogatis LR. Validated instruments for assessing female sexual dysfunction. J Sex Mar Ther 2002;28(Suppl 1):155-64.

21. Baser RE, Li Y, Carter J. Psychometric validation of the Female Sexual Function Index (FSFI) in cancer survivors. Cancer 2012;118:4606-18.

22. Bartula I, Sherman KA. The Female Sexual Functioning Index (FSFI): evaluation of acceptability, reliability, and validity in women with breast cancer. Support Care Cancer 2015;23:2633-41.

23. Derogatis LR, Rosen R, Leiblum S, et al. The female sexual distress scale (FSDS): initial validation of a standardized scale for assessment of sexually related personal distress in women. J Sex Marital Ther 2002;28:317-30.

24. Derogatis L, Clayton A, Lewis-D'Agostino D, et al. Validation of the female sexual distress scale-revised for assessing distress in women with hypoactive sexual desire disorder. J Sex Med 2008;5:357-64.

25. DeRogatis L, Pyke R, McCormack J, et al. Does the female sexual distress scale-revised cover the feelings of women with HSDD? J Sex Med 2011;8:2810-5. 
26. Thirlaway K, Fallowfield L, Cuzick J. The sexual activity questionnaire: a measure of women's sexual functioning. Qual Life Res 1996;5:81-90.

27. Atkins L, Fallowfield LJ. Fallowfield's sexual activity questionnaire in women with without and at risk of cancer. Menopause Int 2007;13:103-9.

28. Vermeulen A, Verdonck L, Kaufman JM. A critical evaluation of simple methods for the estimation of free testosterone in serum. J Clin Endocrinol Metab 1999;84:3666-72.

29. Greene JG. Constructing a standard climacteric scale. Maturitas 1998;29:25-31.

30. Travers C, O'Neill SM, King R, et al. Greene climacteric scale: norms in an Australian population in relation to age and menopausal status. Climacteric 2005;8:56-62.

31. Lewis JE, Hilditch JR, Wong CJ. Further psychometric property development of the menopause-specific quality of life questionnaire and development of a modified version, MENQOL-Intervention questionnaire. Maturitas 2005;50:209-21.

32. Van Dole KB, DeVellis RF, Brown RD, et al. Evaluation of the Menopause-Specific Quality of Life Questionnaire: a factor-analytic approach. Menopause 2012;19:211-5.

33. Radtke JV, Terhorst L, Cohen SM. The Menopause-Specific Quality of Life Questionnaire: psychometric evaluation among breast cancer survivors. Menopause 2011;18:289-95.

34. Buysse DJ, Reynolds CF, Monk TH, et al. The Pittsburgh Sleep Quality Index: a new instrument for psychiatric practice and research. Psychiatry Res 1989;28:193-213.

35. Backhaus J, Junghanns K, Broocks A, et al. Test-retest reliability and validity of the Pittsburgh Sleep Quality Index in primary insomnia. $J$ Psychosom Res 2002;53:737-40.

36. Myers JK, Weissman MM. Use of a self-report symptom scale to detect depression in a community sample. Am J Psychiatry 1980;137:1081-4.

37. Spitzer RL, Kroenke K, Williams JB, et al. A brief measure for assessing generalized anxiety disorder: the GAD-7. Arch Intern Med 2006;166:1092-7.

38. Buckley DI, Fu R, Freeman M, et al. C-reactive protein as a risk factor for coronary heart disease: a systematic review and metaanalyses for the U.S. Preventive Services Task Force. Ann Intern Med 2009;151:483-95.

39. Seibel MJ. Biochemical markers of bone turnover part II: clinical applications in the management of osteoporosis. Clin Biochem Rev 2006;27:123-38.
40. Maclnnis RJ, Cassar C, Nowson CA, et al. Determinants of bone density in 30- to 65-year-old women: a co-twin study. J Bone Miner Res 2003;18:1650-6.

41. Wiegel M, Meston C, Rosen R. The female sexual function index (FSFI): cross-validation and development of clinical cutoff scores. $J$ Sex Marital Ther 2005;31:1-20.

42. West SL, D'Aloisio AA, Agans RP, et al. Prevalence of low sexual desire and hypoactive sexual desire disorder in a nationally representative sample of US women. Arch Intern Med 2008;168:1441-9.

43. Lammerink EAG, de Bock GH, Pascal A, et al. A survey of female sexual functioning in the general Dutch population. J Sex Med 2017;14:937-49.

44. Leiblum SR, Koochaki PE, Rodenberg CA, et al. Hypoactive sexual desire disorder in postmenopausal women: US results from the Women's International Study of Health and Sexuality (WISHeS). Menopause 2006;13:46-56.

45. Challberg J, Ashcroft L, Lalloo F, et al. Menopausal symptoms and bone health in women undertaking risk reducing bilateral salpingooophorectomy: significant bone health issues in those not taking HRT. Br J Cancer 2011;105:22-7.

46. Harris PA, Taylor R, Thielke R, et al. Research electronic data capture (REDCap)--a metadata-driven methodology and workflow process for providing translational research informatics support. J Biomed Inform 2009;42:377-81.

47. Zeger SL, Liang KY, Albert PS. Models for longitudinal data: a generalized estimating equation approach. Biometrics 1988;44:1049-60.

48. Shrier I, Platt RW. Reducing bias through directed acyclic graphs. BMC Med Res Methodol 2008;8:70.

49. Hernán MA, Hernández-Díaz S, Werler MM, et al. Causal knowledge as a prerequisite for confounding evaluation: an application to birth defects epidemiology. Am J Epidemiol 2002;155:176-84.

50. Campfield Bonadies D, Moyer A, Matloff ET. What I wish I'd known before surgery: BRCA carriers perspectives after bilateral salipingooophorectomy. Fam Cancer 2011;10:79-85.

51. Hallowell N, Mackay J, Richards M, et al. High-risk premenopausal women's experiences of undergoing prophylactic oophorectomy: a descriptive study. Genet Test 2004;8:148-56.

52. Firth $\mathrm{C}$, Jacobs $\mathrm{C}$, Evison $\mathrm{M}$, et al. Novel one-stop multidisciplinary follow-up clinic for BRCA1/2 carriers: patient satisfaction and decision making. Psychooncology 2011;20:1301-8.

53. Harmsen MG, Arts-de Jong M, Horstik K, et al. Very high uptake of risk-reducing salpingo-oophorectomy in BRCA1/2 mutation carriers: a single-center experience. Gynecol Oncol 2016;143:113-9. 